УДК 534.833.532

\title{
Применение метода главных компонент к исследованию акустической эмиссии при пластической деформации свинцовых сплавов
}

\author{
Д.С. Салита ${ }^{1}$ В.В. Поляков ${ }^{1,2}$
}

${ }^{1}$ Алтайский государственный университет (Барнаул, Россия)

${ }^{2}$ Институт физики прочности и материаловедения СО РАН (Томск, Россия)

\section{Application of Principal Components Analysis to the Investigation of Acoustic Emission in Plastic Deformation of Lead Alloys}

\author{
D.S. Salita ${ }^{1}$, V.V. Polyakov ${ }^{1,2}$ \\ ${ }^{1}$ Altai State University (Barnaul, Russia) \\ ${ }^{2}$ Institute of Strength Physics and Materials Science of the Siberian Branch \\ of the RAS (Tomsk, Russia)
}

\begin{abstract}
Проведено исследование зависимости акустической эмиссии, формирующейся при статической деформации свинцовых сплавов, от стадий деформационного упрочнения. Установлено существенное изменение информативных характеристик акустико-эмиссионных сигналов при смене стадий деформационного упрочнения, отражавшее эволюцию физических механизмов деформации при нагружении материала. Количественный анализ влияния стадий на акустико-эмиссионные сигналы был проведен с помощью метода главных компонент. При обработке экспериментальных данных весь регистрировавшийся в процессе нагружения сигнал разбивался на малые блоки, каждый из которых отражал поведение материала на соответствующем участке кривой нагружения. Акустическая эмиссия для отдельного блока описывалась с помощью информативных параметров сигнала, отражавших его энергетические и частотные особенности. Проведенная обработка экспериментальных данных позволила провести кластеризацию сигналов в соответствии с выделенными стадиями деформационного упрочнения.
\end{abstract}

Полученные результаты могут быть использованы при акустико-эмиссионном исследовании стадийности процессов пластической деформации в материалах и для диагностики их деформационного поведения.

Ключевые слова: акустическая эмиссия, пластическая деформация, свинцовые сплавы, диагностика материалов.
A study was made of the dependence of acoustic emission, formed during static deformation of lead alloys, on the stages of strain hardening. An essential change in the informative characteristics of acoustic emission signals during the change in the stages of strain hardening, reflecting the evolution of the physical mechanisms of deformation during loading of the material, is established. Quantitative analysis of the effect of stages on acoustic emission signals was carried out using the principal component method. When processing the experimental data, the entire signal recorded during the loading process was divided into small blocks, each of which reflected the behavior of the material in the corresponding portion of the loading curve. Acoustic emission for an individual unit was described using informative signal parameters that reflected its energy and frequency characteristics. The processing of the experimental data made it possible to cluster the signals in accordance with the isolated stages of strain hardening.

The obtained results can be used in the acousticalemission study of the stagedness of the processes of plastic deformation in materials and for the diagnosis of their deformation behavior.

Key words: acoustic emission, plastic deformation, lead alloys, diagnostic materials.

DOI 10.14258/izvasu(2018)4-04

\footnotetext{
*Статья подготовлена при финансовой поддержке РФФИ в рамках проекта №17-08-00914.
} 


\section{Введение}

Исследование особенностей акустической эмиссии, возникающей при механическом нагружении материала, позволяет получить важную информацию о физических механизмах пластической деформации и разрушения $[1,2]$. Существенный интерес представляет анализ акустической эмиссии в свинцовых сплавах, характеризующихся весьма низкой температурой плавления и высокой пластичностью при комнатных температурах [3]. Это делает сплавы на основе свинца важными модельными объектами для изучения деформационных процессов [4-6]. Кроме того, выявление закономерностей пластической деформации этих материалов представляет самостоятельный интерес в связи с их широким использованием в качестве баббитов.

В процессе нагружения наблюдается значительное изменение информативных характеристик акустико-эмиссионных сигналов, отражающее эволюцию физических механизмов деформации и смену доминирующих источников акустического излучения. В то же время в связи со сложностью протекающих процессов и одновременным действием конкурирующих механизмов и источников физическая интерпретация экспериментальных данных достаточно затруднительна [2]. Эффективным подходом к выявлению закономерностей формирования акустической эмиссии является применение новых методов математической обработки регистрируемых сигналов [7-9]. В настоящей работе с помощью проекционных методов анализа многомерных данных описывается связь между стадиями деформационного упрочнения, проходимыми материалом в процессе нагружения, и информативными характеристиками акустической эмиссии, формирующейся в свинцовом сплаве.

\section{Материалы и экспериментальные результаты}

Испытания проводились для образцов свинцового сплава, содержащего добавки легирующих элементов
( $\mathrm{Sb}, \mathrm{Cd}, \mathrm{Ga}, \mathrm{Cu}, \mathrm{Fe})$ с суммарной концентрацией порядка $10 \%$, описанного в [3]. Образцы изготавливались из расплава и подвергались последующей механической обработке. Они имели стандартную форму для испытаний на статическое растяжение с размером рабочей части $50 \times 10 \times 5$ мм.

Нагружение проводилось на установке механических испытаний с постоянной скоростью растяжения. В процессе деформирования одновременно регистрировались абсолютная деформация и приложенная нагрузка, по которым далее рассчитывались истинное напряжение б и истинная (логарифмическая) деформация $\varepsilon$. Для выделения стадий деформационного упрочнения в истинных координатах вычислялся коэффициент деформационного упрочнения $\mathrm{K}=\mathrm{d} \sigma / \mathrm{d} \varepsilon[10]$.

В качестве параметров, характеризующих акустическую эмиссию, измерялись среднеквадратичные значения напряжения $U$, а также скорость счета акустической эмиссии $\dot{N}$. Скорость счета с помощью теоретических моделей механики сплошной среды может быть связана с такими характеристиками, как коэффициенты интенсивности напряжений и параметры трещинообразования, что делает ее удобной дополнительной величиной при интерпретации эксперимента.

Типичный результат механических испытаний представлен на рис. 1 в виде деформационной кривой $\sigma(\varepsilon)$ и зависимости К $(\varepsilon)$. Как следует из рис. 1 , по изменению коэффициента деформационного упрочнения можно выделить четыре стадии деформационного упрочнения: очень короткая стадия линейного упрочнения I, две стадии параболического упрочнения II и III, описывающие пластическое течение свинцового образца при невысокой нагрузке, и стадия предразрушения и разрушения IV.

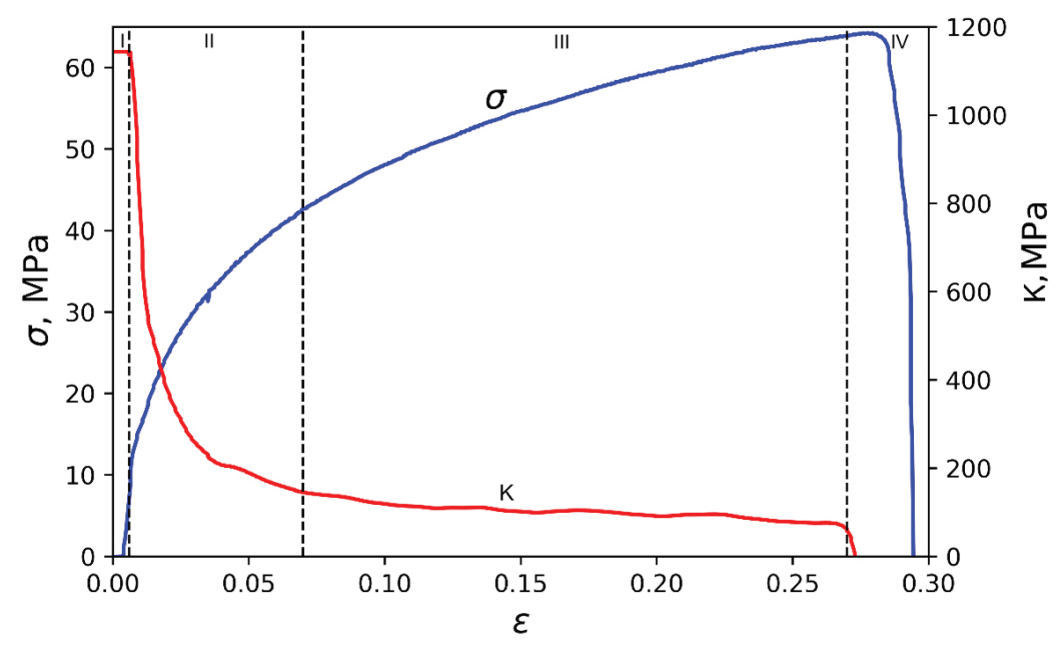

Рис. 1. Деформационное упрочнение свинцового сплава.

I, II, III, IV — стадии деформационного упрочнения 
Типичный вид скорости счета акустической эмиссии в зависимости от величины $\varepsilon$ приведен на рис. 2 . Из рис. 2 видно, что наблюдается значительное изменение вида кривой $\dot{N}(\varepsilon)$, описывающей акустико-эмиссионные сигналы, при переходе с ростом деформации от одной стадии упрочнения к другой. На стадии I акустическая эмиссия практически не проявляется. На стадии II формируется серия вы- сокоамплитудных пиков. Появление этих пиков может быть связано с движением и выходом на поверхность мощных дислокационных потоков. Наиболее длительной стадии пластического течения III соответствует участок осциллирующей акустической эмиссии с постепенно спадающей к концу стадий амплитудой осцилляций. На заключительной стадии IV сигналы акустической эмиссии незначительны.

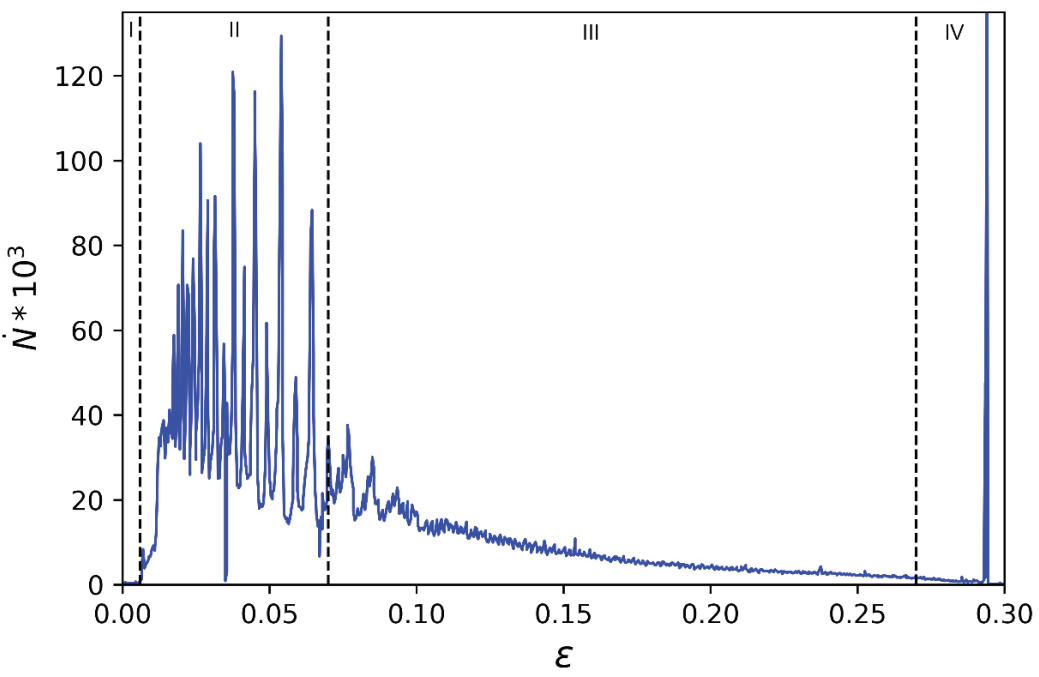

Рис. 2. Акустическая эмиссия при нагружении свинцового сплава. I, II, III, IV - стадии деформационного упрочнения

\section{Применение метода главных компонент}

Результаты рис. 2 показывают, что характеристики акустической эмиссии отражают физические процессы, протекающие в материале на соответствующей стадии деформационного упрочнения. Это может позволить определять проходимую нагружаемым материалом стадию по виду акустико-эмиссионных сигналов. Для установления количественной связи между информативными характеристиками и стадиями регистрировавшиеся сигналы были обработаны с помощью метода главных компонент $[11,12]$. Этот метод позволяет провести кластеризацию экспериментальных данных, то есть разделить акустико-эмиссионные сигналы на группы с близкими свойствами, отвечавшими определенной стадии упрочнения.

Применение метода главных компонент проводилось следующим образом. Весь зарегистрированный в процессе нагружения сигнал разбивался на малые блоки длительностью 1 с., каждый такой блок отражал поведение материала на соответствующем малом участке кривой нагружения. Акустическая эмиссия для отдельного блока описывалась с помощью совокупности информативных параметров сигнала, формировавших вектор признаков. В качестве этих параметров использовались амплитудные характеристики - среднеквадратичное значение на- пряжения $\mathrm{U}$, максимальное значение $\mathrm{U}_{\mathrm{m}}$, пик-фактор $\mathrm{U}_{\mathrm{p}}=\mathrm{U}_{\mathrm{m}} / \bar{U}(\bar{U}-$ среднее по блоку значение $)$, фактор формы $U_{\mathrm{f}}=\bar{U} / \mathrm{U}$, а также полное число импульсов N. Эта совокупность параметров рассматривалась как точка в многомерном пространстве главных компонент. В результате применения метода исходные данные представлялись в виде геометрической проекции на координатные плоскости, образованные первыми главными осями. Точки, описывавшие блоки с близкими физическими характеристиками, располагались вблизи друг от друга в одной области и образовывали кластеры.

Результаты расчетов представлены на рис. 3 для двух первых главных компонент ГК1 и ГК2. Точки II, III, IV описывали отдельные блоки, приходящиеся на стадии деформационного упрочнения II, III, IV соответственно. Как видно из рис. 3, все точки разделились на кластеры, соответствующие стадиям деформационного упрочнения. Это разделение обеспечивалось главной компонентой PC1, описывавшей энергетические и частотные характеристики исходных сигналов. Таким образом, проведенная обработка позволила установить количественную связь между характеристиками акустико-эмиссионных сигналов и стадиями деформационного упрочнения материала. 


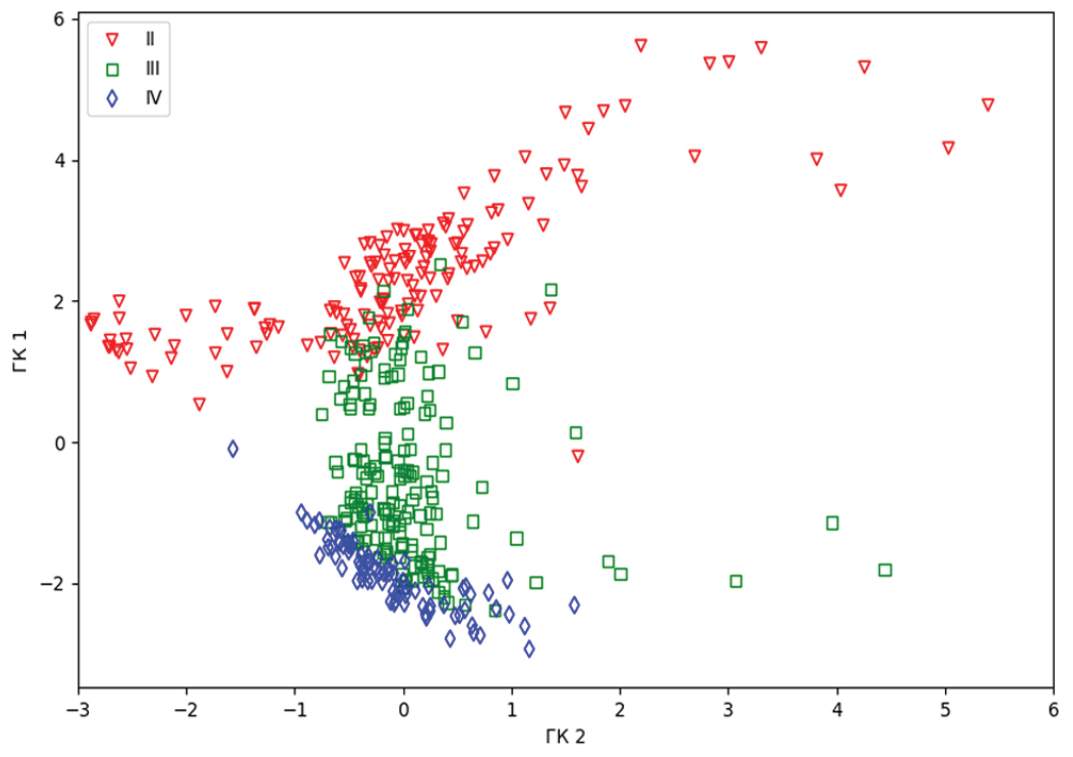

Рис. 3. Проекция характеристик акустико-эмиссионного сигнала на плоскость первых главных компонент. II, III, IV - стадии деформационного упрочнения

\section{Заключение}

В работе описана зависимость акустической эмиссии, формирующейся в свинцовом сплаве при нагружении, от стадии деформационного упрочнения. Рассмотрены информативные характеристики, описывавшие энергетические и частотные особенности сигналов акустической эмиссии. Для количественного анализа влияния стадий деформационного упроч- нения на акустическую эмиссию использован метод главных компонент, обеспечивший кластеризацию экспериментальных акустико-эмиссионных сигналов в соответствии с выделенными стадиями.

Предложенный подход может быть применен при акустико-эмиссионном исследовании стадийности процессов пластической деформации и для диагностики деформационного поведения материалов.

\section{Библиографический список}

1. Буйло С.И. Физико-механические и статистические аспекты повышения достоверности результатов акустико-эмиссионного контроля и диагностики. - Ростов н/Д., 2008.

2. Егоров А.В., Поляков В.В. Применение метода акустической эмиссии к исследованию деформационного поведения структурно-неоднородных материалов. - Барнаул, 2008.

3. Салита Д.С., Поляков В.В. Влияние структуры свинцового сплава на акустическую эмиссию при пластической деформации и разрушении // Известия АлтГУ. — 2017. № 1 .

4. Панин В.Е., Егорушкин В.Е., Елсукова Т.Ф. Физическая мезомеханика зернограничного скольжения в деформируемом поликристалле // Физическая мезомеханика. 2011. - T. 14.

5. Панин В.Е., Елсукова Т.Ф., Панин А.В., Кузина О.Ю., Кузнецов П.В. Мезоскопические структурные уровни деформации в поверхностных слоях и характер усталостного разрушения поликристаллов при знакопеременном изги- бе. Ч. І. Мезоскопическая субструктура // Физическая мезомеханика. - 2004. - Т. 7.

6. Елсукова Т.Ф., Новоселова Е.М., Караваева В.В., Ангелова Г.В. Стадии высокотемпературной ползучести поликристаллов свинца как эволюция структурных уровней пластической деформации // Физическая мезомеханика. $-2000 .-$ Т. 3.

7. Дмитриев А.А., Поляков В.В., Лепендин А.А. Исследование пластической деформации алюминиевых сплавов с использованием вейвлет-преобразований сигналов акустической эмиссии. Письма о материалах. - 2018. - Т. 8, вып. 1.

8. Ferreira D.B.B., Da Silva R.R., Rebello J.M.A., Siqueira M.H.S. Failure mechanism characterisation in composite materials using spectral analysis and the wavelet transform of acoustic emission signals // Insight. - 2004. - Vol. 46, issue 5 .

9. Shahri M.N., Jalal Yousefi J., Fotouhi M., Najfabadi M.A. Damage evaluation of composite materials using acoustic 
emission features and Hilbert transform // Journal of Composite Materials. - 2015. - Vol. 50, issue 14.

10. Баранникова С.А., Данилов В.И., Зуев Л.Б. Локализация пластической деформации в моно- и поликристаллах сплава $\mathrm{Fe}-3 \% \mathrm{Si}$ при растяжении // Журнал технической физики. - 2004. - Т. 74, вып. 10.
11. Esbensen K.H. Multivariate Data Analysis - In Practice. CAMO Process AS. - Esbjerg, 2002.

12. Sophian A., Tian G.Y., Taylor D., Rudlin J. A feature extraction technique based on principal component analysis for pulsed Eddy current NDT // NDT E Int. — 2003. - Vol. 36. 